Contrôles : frontières, identités. Les enjeux autour de l'immigration et de l'asile

\title{
Immigration, race et sécurité à la frontière mexicano-californienne
}

\section{Randy Willoughby}

\section{(2) OpenEdition}

\section{Journals}

Édition électronique

URL : http://journals.openedition.org/conflits/371

DOI : $10.4000 /$ conflits.371

ISSN : $1777-5345$

Éditeur :

CCLS - Centre d'études sur les conflits lilberté et sécurité, L'Harmattan

Édition imprimée

Date de publication : 15 octobre 1997

ISSN : 1157-996X

Référence électronique

Randy Willoughby, «Immigration, race et sécurité à la frontière mexicano-californienne », Cultures \&

Conflits [En ligne], 26-27 | automne 1997, mis en ligne le 15 mars 2006, consulté le 30 mars 2021

URL : http://journals.openedition.org/conflits/371 ; DOI : https://doi.org/10.4000/conflits.371

Ce document a été généré automatiquement le 30 mars 2021.

Creative Commons License 


\title{
Immigration, race et sécurité à la frontière mexicano-californienne
}

\author{
Randy Willoughby
}

Randy WILLOUGHBY ${ }^{1}$

" Partout dans le monde, il y a des contradictions. S'il n'y en avait pas, il n'y aurait pas de monde. " Mao Zédong ${ }^{2}$

Introduction Les politiques d'immigration adoptées actuellement par les pays occidentaux comportent tant de paradoxes et de contradictions que nous pourrions donner raison au Grand Timonier, ne serait-ce que pour son insistance judicieuse sur la dialectique. En paraphrasant le Grand Leader, nous pourrions dire que " s'il n'y avait pas de contradictions il n'y aurait pas d'immigration ". Cela a été déjà observé par de nombreux auteurs appartenant à des disciplines diverses. Ainsi, un politologue parle de la " politique boomerang " d'un important parti politique mexicain (le PRD de Cardenas) qui cherche à accroître son influence au Mexique en organisant des campagnes à Los Angeles car " la politique intérieure n'est plus intérieure "3. En analysant le lien entre l'idéologie de l'immigration et l'économie, un sociologue pose l'éternelle question de l'oeuf et de la poule et montre la " contradiction entre les politiques de jure et de facto suite à la réforme de la législation américaine en matière d'immigration en 1986 " 4 . Un économiste cite de manière prosaïque " "le paradoxe libéral", selon lequel les démocraties industrielles encouragent les pays en voie de développement à adopter le libre-échange et l'investissement et ensuite... se plaignent que des travailleurs originaires de ces pays traversent leurs frontières "5. Et enfin, un romancier commence son récit sur l'immigration par " la chute angélico-démoniaque " de deux Indiens dans la Manche - l'un, totalement assimilable, l'autre, irrespectueux et cherchant à " tropicaliser " Londres, le premier poussant des cornes diaboliques et le second se parant d'une auréole angélique ${ }^{6}$. Dans cet article, nous tâcherons de montrer que la contradiction occupe une place centrale dans l'histoire de l'immigration américaine. Les débats classiques du style Oxford et les présentations populaires de " points de vues opposés " obscurcissent le caractère compliqué et souvent dialectique de la dynamique de l'immigration. Leur prétention à élaborer une approche particulière, 
consistante et cohérente s'avère plutôt trompeuse. Nous essaierons de le démontrer en nous référant aux dilemmes, aux effets pervers, aux ironies et aux autres " contradictions " du débat actuel sur l'immigration aux États-Unis et en insistant plus particulièrement sur le référendum californien portant sur la mesure qui vise à priver les immigrants clandestins d'accès aux divers services publics (Proposition 187). Considérons, par exemple, l'importance accordée depuis 1965 au regroupement familial dans la sélection des immigrants, qui a donné lieu en 1993 à l'admission de 482000 personnes, contre 147000 admises pour leur qualification professionnelle et 127000 en tant que réfugiés politiques, et comparons-la avec la réputation des États-Unis d'être à la fois le pays le plus profondément individualiste et capitaliste du monde et une terre d'" asile pour l'humanité " (selon Thomas Paine). Rappelons la campagne onéreuse qu'avait menée Michael Huffington pour gagner le siège californien au Sénat, campagne qui s'était focalisée sur le problème de l'immigration clandestine et avait abouti à la révélation que Huffington lui-même avait employé un clandestin (tout comme les deux candidats soutenus par Clinton pour le poste du Procureur général ainsi que le rival de Huffington à l'élection sénatoriale). Rappelons comment Viva Los Mojados, un corrido mexicain, défie les préjugés et la loi : " la loi n'aime pas les wetbacks ${ }^{7}$ car ils sont clandestins et ne parlent pas l'anglais, mais le problème peut s'arranger en épousant une " gringuita ", en régularisant ainsi sa situation et, puis, en divorçant d'elle ". Prenons enfin la construction d'une redoutable clôture en acier entre San Diego et Tijuana l'année même (1989) de la destruction du Mur de Berlin et de l'adoption du Traité de l'ALENA par la Chambre des Représentants, avec le même pourcentage des voix (59\% contre 41\%) que celui obtenu lors de l'adoption de la Proposition 187 par les électeurs californiens (décrite comme la preuve la plus récente du " paradoxe libéral " de P. Martin et M. Miller) ${ }^{8}$. Loin de se limiter à la seule approche dialectique, cet essai adopte l'aspect multidimensionnel du fameux discours prononcé par le Grand Timonier en 1956 sur " les dix grandes relations ". Il propose de construire un cadre général reliant les perspectives historique, définitionnelle, économique, politique, raciale, internationale et sécuritaire en matière d'immigration. Nous nous focaliserons plus particulièrement sur les questions relatives à la race et à la sécurité ou, plutôt, sur ce que Gunnar Myrdal appelait " le dilemme américain ", en se référant au " problème noir et à la démocratie moderne ", ainsi qu'à ce que les spécialistes des relations internationales désignent par " dilemme sécuritaire ", c'est-à-dire l'idée selon laquelle l'effort accompli en vue de renforcer le sentiment de sécurité dans la population ne fait que renforcer la provocation. Malgré le risque de paraître exagéré, nous estimons que ces deux dynamiques offrent au débat actuel sur l'immigration, tant aux États-Unis que dans d'autres pays, une qualité particulière et un caractère passionnant. Les idéologies de la race et de la sécurité ont été à l'origine des plus grandes mobilisations de l'histoire américaine - la Guerre Civile au XIXe siècle, les deux guerres mondiales et la Guerre Froide au XXe siècle. Avec la fin de la bipolarité, ces idéologies ont rejoint le débat sur l'immigration non seulement en s'entremêlant avec les rhétoriques professionnelles et académiques, mais aussi en se renforçant par des images hollywoodiennes très réussies $\mathrm{du}$ pandémonium culturel (notamment Bladerunner, qui décrit Los Angeles en 2 019) et le djihad nucléaire (le film d'action de Schwarzenegger sur la côte Est True Lies). Incontestablement, l'élément racial est loin d'être déterminant en la matière et paraît plus implicite qu'explicite; l'aspect sécuritaire est plus interne qu'externe et les menaces se présentent différemment selon les pays et les groupes d'immigrants. La campagne contre l'immigration clandestine de 
la Proposition 187 du référendum californien s'est lentement transformée en une " Initiative pour les droits civiques ", atteignant ainsi un momentum fortuit. Et la perception des liens entre les causes du nouveau désordre mondial, à savoir le crime organisé, le trafic de stupéfiants, le terrorisme et l'immigration clandestine, désignée de manière critique par Didier Bigo comme un " continuum de menaces "9, a été renforcée aux États-Unis au cours de ces dernières années suite aux violents incidents déclenchés au Mexique ainsi qu'à l'attentat contre le World Trade Center à New York. Finalement, on peut dire que la politique américaine d'immigration, qui était normalement du ressort de l'agrobusiness, des syndicats, des économistes et des avocats spécialisés, voire même des curateurs des immigrés d'Ellis Island, est passé, du moins provisoirement, de la politique habituelle et pragmatique de défense des intérêts privés et de la régulation à une politique idéologiquement bien chargée de redistributions et de conflits. L'histoire " Bon débarras des mauvais débris. "10 L'actuelle politique d'immigration des Etats-Unis repose sur la loi sur l'immigration et la nationalité de 1952 ainsi que sur des amendements ultérieurs. Même si cette législation a donné lieu à une multiplication des possibilités d'immigration aux États-Unis, notamment en supprimant les quotas nationaux en 1965, en élargissant la définition des réfugiés en 1980, en prévoyant un programme généreux d'amnistie pour les travailleurs clandestins en 1986 et en élargissant les limites numériques de l'immigration en 1990, un de ses effets immédiats aura été de fournir au Procureur général une base légale (sur fond moral et idéologique) pour interdire à Charlie Chaplin de rentrer aux États-Unis après son voyage à Londres pour la première mondiale de Limelight. Après la statue de la Liberté, il n'y a probablement pas d'autre image plus puissante sur la " nation d'immigrants " que le court-métrage L'émigrant, tourné par Chaplin en 1917 et repris ensuite par Louis Malle dans Au Revoir les Enfants, qui traite de la persécution des enfants juifs par la Gestapo dans la France occupée. L'évolution de Chaplin, qui est passé du statut d'immigrant bienvenu à celui de fauteur de troubles dénigré, illustre de manière hautement symbolique la contradiction la plus évidente de l'histoire américaine en matière d'immigration, c'est-à-dire le passage cyclique de l'admission à l'exclusion. En Californie, la figure qui représente cette contradiction est Denis Kearney, qui " était devenu en 1878 l'individu le plus redouté de la côte Pacifique "11. Né en Irlande en 1847, installé à San Francisco en 1872, devenu secrétaire du Workingman's Party en 1877, Kearney, en tant que membre de la " Pickhandle Brigade ", a aidé la police à réprimer les émeutes contre les coolies et a prononcé des discours enflammés où il clamait haut et fort: " les Chinois doivent partir ". L'ouvrage de Bill Ong Hing sur l'immigration asiatique aux États-Unis montre bien que ce changement d'attitude était loin d'être exceptionnel à la fin du XIXe siècle : en 1868, les Californiens avaient accueilli avec " des fanfares et des manifestations de joie " la conclusion du Traité de Burlingame entre les États-Unis et la Chine parce que cette dernière acceptait de mettre fin au strict contrôle qu'elle exerçait sur l'émigration de ses ressortissants, mais, lors du référendum de $1879,94 \%$ des électeurs californiens votèrent contre la présence d'immigrants chinois, préparant ainsi le terrain à l'adoption en 1882 de la loi sur l'exclusion des Chinois. L'adoption de la Proposition 187 a révélé un élément de l'esprit Kearney et a renversé la règle d'or dans la mesure où, selon un sondage effectué par le Los Angeles Times, 54\% des immigrants de première génération ayant participé au référendum ont voté " oui " (ils représentaient $10 \%$ de l'ensemble des électeurs). Cette dynamique d'ouverture et de fermeture des portes a donné lieu à des fluctuations profondes des flux migratoires vers les États-Unis au cours du siècle dernier et a abouti 
à présent à une position plus ou moins intermédiaire. De 1870 à 1930, arrivèrent en permanence entre 2,8 et 8,8 millions d'immigrants par décennie (atteignant une moyenne de plus de 5 millions), tandis que de 1930 à 1970 le niveau était entre 0,5 et 3,3 millions d'immigrants par décennie (atteignant une moyenne de moins de 2 millions) ${ }^{12}$. Les niveaux les plus récents de l'immigration tendent à renouer avec des sommets historiques calculés en termes absolus, en moyennes historiques ou en termes de pourcentage de population. Si nous comptons seulement les immigrés légaux, nous constatons qu'il y en avait environ un million dans les années quarante, deux millions et demi dans les années cinquante, trois millions et demi dans les années soixante, quatre millions et demi dans les années soixante-dix, sept millions et demi dans les années quatre-vingt et treize millions et demi dans les années quatre-vingt-dix (vu qu'entre 1990 et 1993 il y a eu cinq millions et demi d'immigrants). Si nous utilisons un mode de calcul différent, nous constatons que $15 \%$ de la population était d'origine étrangère entre 1870 et 1920 , contre $8 \%$ en $1990^{13}$. À présent, il semble que le pendule oscille lentement mais sans faille vers le renforcement des restrictions. Le pourcentage de l'opinion publique souhaitant la réduction du niveau de l'immigration est passé dans les trois dernières décennies de 33\% en 1965 à $42 \%$ en 1977, à $49 \%$ en 1986 et à $61 \%$ en 1993. Néanmoins, ce qui est frappant dans la dialectique actuelle des États-Unis ce n'est pas tant la campagne pour l'adoption de mesures restrictives, dont le discours et l'inspiration sont selon Livi Bacci plus ou moins similaires à ceux des pays occidentaux prospères, que la fin d'une idéologie positive qui associait l'immigration au succès américain. En d'autres termes, surtout sur le plan du mythe, la plupart des pays du monde ont moins une dynamique pendulaire en matière d'immigration qu'une simple présomption à sens unique contre elle. Aux États-Unis, en 1993, une couverture du Newsweek Magazine montrait un dessin représentant la statue de la Liberté recouverte jusqu'au cou par un raz-de-marée de boat-people, tandis qu'au même moment le Time Magazine publiait en couverture, de son numéro spécial consacré à l'immigration, un visage féminin séduisant et exotique, le " nouveau visage de l'Amérique ", une image virtuelle fabriquée par ordinateur et montrant comment les immigrés modèlent la première société multiculturelle $\mathrm{du}$ monde. Pour montrer combien cette femme imaginaire, composée à partir de traits sud européens (35\%), anglo-saxons (15\%), moyen-orientaux $(17,5 \%)$, africains $(17,5 \%)$, asiatiques $(7,5 \%)$ et hispaniques $(7,5 \%)$, était attirante, le directeur de la rédaction avait dit : " Pendant qu'on voyait apparaître sur l'écran de l'ordinateur l'image de notre nouvelle Eve, plusieurs membres de la rédaction en étaient déjà tombés amoureux ". Quant au New York Times, il consacrait sa page éditoriale à Tijuana et publiait une image qui battait en brèche les préjugés sur les clandestins: on voyait les quatre politiciens importants de la côte Est (Kemp, Cuomo, Giuliani et Bennett) qui étaient d'abord entassés dans le coffre d'une voiture du côté de Tijuana de la frontière mais qui, ensuite, étaient éjectés de la voiture, car " ils avaient fait des déclarations qui, dans le temps, étaient considérées comme convenues aux États-Unis mais qui, maintenant, sont devenues terriblement démodées, voire même dangereuses pour les politiciens "14. Dans la même lignée, citons également le résolument conservateur San Diego Union-Tribune, qui non seulement avait endossé l'opposition à la Proposition 187 en 1994 mais avait aussi commencé la nouvelle année avec un spécial cinq pages de Business Perspectives défendant l'immigration légale; la série d'articles était intitulée: " Il y a encore des possibilités de CROISSANCE: les immigrés déterminés prouvent que le rêve américain est vivant et bien portant "15. Définition En 1908, à San Andres de Sotavento, le gouverneur, le général Miguel Marino 
Torralvo, publie l'ordonnance sur les compagnies de pétrole opérant sur la côte colombienne : " Les Indiens n'existent pas... S'ils existaient ils seraient des clandestins. "16 Généralement, les débats sur l'immigration clandestine fournissent des chiffres mais n'éclaircissent que très rarement les présuppositions et les définitions sous-jacentes. Par exemple, pour obtenir plus de soutien à son paquet d'aide du sauvetage du peso mexicain, le Président Clinton avait avancé qu'une détérioration éventuelle de la situation économique $d u$ Mexique entraînerait l'arrivée d'un demi million d'immigrants clandestins dans les États du Texas et de Californie en 1995. Une estimation que l'Administration s'était empressée de corriger quelques jours plus tard. Ainsi, un haut fonctionnaire du ministère du Trésor avait déclaré que la hausse éventuelle ne serait plutôt que d'environ 40000 (car le chiffre initial comprenait, entre autres, des personnes qui n'entraient aux États-Unis que pour faire de brèves courses) mais qu'elle ne tenait pas compte de l'impact du renforcement des opérations menées par la Border Patrol, telles que Gatekeeper à San Diego et Hold the Line à El Paso ${ }^{17}$. En fait, même ces chiffres approximatifs étaient problématiques car ils s'appuyaient sur une équation implicite, selon laquelle chaque dévaluation du peso donnerait lieu à une augmentation de l'immigration clandestine, une équation qui, si elle s'était avérée juste, aurait entraîné l'émigration de tous les Mexicains aux États-Unis entre 1976 et 1994, lors de la dépréciation du peso de 12,5/1 à 3.000/1. Selon les estimations de l'INS, en 1994 il y avait 4 millions d'immigrants clandestins aux Etats-Unis (la hausse annuelle atteignant une moyenne de 300000 personnes), dont environ $40 \%$ se trouvaient en Californie et près de $60 \%$ étaient d'origine mexicaine ${ }^{18}$. Le pourcentage des clandestins qui traversent illégalement la frontière et de ceux qui restent après l'expiration de leur visa était pratiquement le même. Bien évidemment, tous ces chiffres ne sont que des estimations qui font l'objet de nombreuses complications méthodologiques et donnent lieu à des résultats contestés. En effet, étant donné l'énorme volume de personnes traversant chaque année les ports d'entrée des États-Unis, la facilité de se procurer de faux documents, l'étendue de la frontière américano-mexicaine et l'ouverture relative de la frontière américano-canadienne, les estimations peuvent varier considérablement suivant les présuppositions qui les sous-tendent. Dans les ports d'entrée de San Diego par exemple, qui sont les plus actifs du pays, voire du monde entier, les douanes et l'INS enregistrent 56 millions de passages légaux par an (environ un toutes les trente secondes). Dans tout le district de San Diego, l'INS a enregistré, en 1994, 91 millions d'entrées, dont $177000(0,02 \%)$ ont fait l'objet de refoulement vers le Mexique $^{19}$. En dehors des ports d'entrée, jusqu'aux opérations de blocage de la frontière à El Paso et à San Diego, la Border Patrol a arrêté plus d'un million de personnes qui essayaient de traverser la frontière clandestinement. Par exemple, dans le district de New York, l'INS contrôle environ 22 millions de visiteurs par an et estime que moins d'un pour cent restent au pays après l'expiration de leur visa ${ }^{20}$. Au niveau national, le recensement de 1980 a enregistré près de deux millions de clandestins et a estimé qu'il y en avait presque six millions dans tout le pays. Selon l'avis de l'Urban Institute, les estimations de l'INS en matière d'immigration clandestine au niveau national restent jusqu'à présent les plus fiables ${ }^{21}$. Par contre, les estimations au niveau des États sont sujettes à caution car elles se fondent sur des informations liées aux régularisations effectuées par l'IRCA et, par conséquent, accusent un retard de nombreuses années. Ainsi, les estimations concernant l'État de Californie continuent-elles de varier considérablement ; l'Urban Institute avance, par exemple, qu'il y a 307000 enfants en situation irrégulière dans les écoles californiennes, tandis que l'État de Californie 
affirme qu'il y en a 392000 - l'écart étant d'environ 25\%. De même, les estimations varient lorsqu'elles se réfèrent au nombre des clandestins arrêtés pour crimes et incarcérés dans des prisons publiques. Selon un rapport présenté par l'Assemblée californienne, les immigrés clandestins représentent $22 \%$ des personnes arrêtées pour crimes dans le comté de San Diego en 1993 (ce taux passant de 3\% à Coronado à 35\% à Escondido) ${ }^{22}$. Il est en outre affirmé que $33 \%$ des clandestins possèdent de faux documents. Lors d'une audition, ces estimations ont pourtant été contestées comme étant systématiquement et profondément exagérées et il a été prouvé que le taux d'arrestation des clandestins pour crimes n'avait pas sensiblement changé depuis 1989, lorsqu'une étude menée de manière plus rigoureuse par SANDAG avait abouti à un taux entre 12 et $15 \%{ }^{23}$. Sans mentionner les machinations politiques qui viennent modifier certains résultats, nous pouvons dire que ces difficultés méthodologiques peuvent à la fin apparaître moins significatives pour le débat sur l'immigration clandestine venant du Mexique que les définitions empruntées. J. Passel et M. Fix ont bien raison quand ils affirment que " la politique américaine d'immigration doit être considérée comme composée non pas d'un mais de trois ensembles de règles bien distincts, régissant respectivement l'immigration légale, les admissions pour motif humanitaire et le contrôle de l'immigration clandestine. Cette distinction est importante car chaque catégorie est régie par une législation différente, dépend de différents réseaux de bureaucrates, est guidée par des objectifs différents et s'applique à des immigrés qui ont des caractéristiques bien différentes "24. Cette approche prudente est appropriée étant donné les conceptions erronées, largement répandues dans l'opinion publique, selon lesquelles, d'une part, la majorité des immigrés aux États-Unis serait en situation irrégulière (selon l'INS ils sont $30 \%$ ), et d'origine mexicaine (40\%), et, d'autre part, les réfugiés ne seraient en fait que des migrants économiques. Ces conceptions populaires sont renforcées par la polémique selon laquelle " si les dirigeants ne travaillent qu'à stopper les entrées irrégulières, cela permettra à l'immigration future de mettre le fardeau sur le dos des États-Unis en quatre ans au lieu de trois comme c'est le cas actuellement "25. Du comté de Suffolk au Capitole, les politiciens ont envisagé des résolutions qui vont plus loin que la Proposition 187, en proposant de priver non seulement les clandestins mais aussi les migrants légaux de l'accès aux soins médicaux gratuits et aux diverses prestations sociales. Ceux qui sont pour l'adoption de mesures restrictives et sévères à l'encontre de tous les immigrants ne sont pas les seuls à créer des amalgames en matière de définition entre immigration légale, immigration clandestine et autres types d'immigration. Certains, en particulier les militants de la communauté chicano/mexicaine-américaine, refusent l'attribution du label d'immigration au mouvement Nord/Sud des Latinos, de sorte que le migrant texan dans un roman de Tomas Rivera, And the Earth Did Not Devour Him, et le migrant mexicain dans un film de Jack Nicholson, The Border, soient non seulement des gens de passage mais des gens de même racine. Le Corrido Pensilvanio parle des migrants mexicains qui vont en Pennsylvanie et en Virginie de l'Ouest en disant qu'ils viennent de Fort Worth ; le romancier Arturo Islas se plaint de tous ces critiques littéraires qui " se trompent car ils ne veulent pas comprendre la distinction bien nette que je fais entre émigrants et immigrants ". Bref, non seulement les programmes politiques accompagnés de campagnes de brouillage de définitions sont diamétralement opposés, mais ces deux défis lancés à la délimitation nette et catégorique de J. Passel et M. Fix sont historiquement et analytiquement importants. L'immigration mexicaine vers les États-Unis a atteint son momentum initial à la fin du XIXe siècle, assez tard pour 
diminuer l'impact de la célèbre phrase que les militants ne cessent de rappeler : " ce ne sont pas les Mexicains qui ont traversé la frontière, c'est la frontière qui les a traversés ", mais assez tôt pour qu'on puisse avancer que c'était l'adoption de textes législatifs et administratifs vers le tournant du siècle qui avait donné lieu à la création de " la catégorie d'immigrés clandestins à partir d'un flux préexistant et préétabli "26. A. Portes explique que, jusqu'au tournant du siècle, les Mexicains traversaient librement la frontière américaine, en ne payant que pour la traversée du Rio Bravo ; " à partir de 1917, ils devaient fournir deux actes de naissance, un certificat de bonne conduite, un certificat médical, des preuves qu'ils ne deviendraient pas une charge publique, une somme de dix dollars pour le visa et de huit dollars pour la taxe d'entrée ". Dans son étude du Programme Bracero, mis en oeuvre de 1942 à 1964, Garcia y Griego montre comment le flux d'immigration clandestine avait été provoqué par des raisons administratives plutôt que démographiques. Par exemple, dans les années cinquante, la baisse rapide du nombre des arrestations de clandestins, passant de 1,1 million en 1954 à 0,25 en 1955 et à 0,07 en 1957, était étroitement liée au recrutement des braceros à la frontière, dont le nombre était passé d'environ 2 millions par an avant 1954 à 3 millions en 1954 et à 4 millions en 1955. En effet, durant l'opération Wetback en 1954, il arrivait souvent que les travailleurs mexicains fussent expulsés des États-Unis et recrutés le même jour par des employeurs américains ${ }^{27}$. D'autre part, à une époque plus récente, l'administration a fait preuve d'une tolérance en faveur de la régularisation des clandestins et de la transformation de leur statut de bracero en résident permanent/ citoyen. La décision de 1987 de la Cour Suprême sur l'affaire Plyler v. Doe se réfère à un arrêt de 1978 de la Cour du district, où il était cité : " sous les lois et les pratiques en vigueur, l'étranger en situation irrégulière d'aujourd'hui peut très bien devenir l'étranger en situation régulière de demain ". Elle se réfère également à un témoignage d'un expert cité à la barre, selon lequel " 50 à $60 \%$ des migrants légaux d'aujourd'hui étaient auparavant des clandestins ". En 1986, l'IRCA - Immigration Reform and Control Act - a préparé le terrain pour la régularisation d'environ trois millions de clandestins. De plus, pendant la première moitié des années quatre-vingt-dix, 250000 étrangers qui n'avaient pas quitté le pays après l'expiration de leur visa sont devenus résidents en se mariant, en trouvant du travail ou grâce à la loterie de visas ${ }^{28}$. Et finalement, suite à la déclaration de l'administration Clinton relative à l'arrêt de la protection temporaire des réfugiés salvadoriens à partir de janvier 1995, le Commissaire de l'INS a expliqué que leur droit de rester et de travailler aux États-Unis serait automatiquement prolongé d'au moins neuf mois, qu'ils seraient informés de leur droit de déposer une demande d'asile politique et que beaucoup seraient susceptibles d'obtenir le statut de résident ou la nationalité américaine puisqu'ils avaient déjà résidé au pays pendant plus de sept ans. Economie " Un quantificateur fanatique du XVIe siècle ... a estimé que le poids d'une balle de $103 / 4$ inches serait de 61 livres, 1 once, 2 drachmes, 1 scrupule et 15 685644/1414944 grains "29. Les calculs économiques sur l'importance de l'immigration semblent contenir un écart étonnant entre la précision individuelle et l'indétermination collective. Selon les estimations faites respectivement par SANDAG (1989) et par L. Rea et R. Parker ${ }^{30}$, le coût annuel des clandestins pour la justice criminelle de Californie serait respectivement de 1522743499 et de 151220101 \$. Bien que cette différence reflète en partie les modifications survenues au fil des ans sur le nombre d'étrangers en situation irrégulière incarcérés dans des prisons publiques, qui est passé de 5600 (ou 7\%) en 1988 à 20147 (ou 16\%) en 1994¹, l'écart entre ces deux estimations est si grand qu'il ne saurait être uniquement attribué aux modifications 
précitées. En ce qui concerne l'impact fiscal des migrants, Donald Huddle, de l'Université de Rice, a estimé qu'ils coûtaient au gouvernement américain 40 milliards de dollars par $a^{32}$, tandis que J. Passel et M. Fix ont estimé que le gouvernement gagne 25-30 milliards de dollars par an. Ces derniers ont affirmé que " selon des estimations qui s'appuient sur une variété de sources et de modes d'investigation, tous les immigrants arrivés aux États-Unis après 1970 paient des impôts de 70 milliards de dollars, à savoir 25-30 milliards de dollars de plus qu'ils ne dépensent en services publics "33. Comme il a été souligné plus haut, ces différences reflètent la variété des choix méthodologiques. Par exemple, la prise en considération ou non des coûts moyens ou marginaux nécessite un certain jugement qui pourrait introduire des modes de calcul différents (quoique, comme le signale G. Borjas, on ne peut raisonnablement supposer que les coûts marginaux soient nuls). Les présupposés viennent du côté conservateur, comme l'illustre l'affirmation de D. Huddle, selon laquelle l'entrée de six immigrants aux États-Unis provoque le chômage d'un citoyen américain ${ }^{34}$. Du côté libéral, W. Cornelius et P. Martin avancent l'idée que l'absence d'accélération de l'immigration de l'Europe du Sud vers l'Europe du Nord en raison de la Communauté européenne pourrait être un des facteurs présageant une hausse limitée à court et à moyen terme de l'immigration mexicaine vers les États-Unis en raison de l'ALENA. Certes, rien de tout cela ne signifie que les informations de nature économique soient inutiles. G. Borjas, par exemple, prouve de manière convaincante que, dans la distribution du Welfare, entre 1970 et 1990, les ménages de migrants sont passés de la sous représentation (ils constituaient 6,8\% de l'ensemble des ménages et recevaient $6,7 \%$ des prestations sociales) à la surreprésentation ( $8,4 \%$ des ménages et $13,1 \%$ des prestations) ${ }^{35}$. Il semble pourtant que, d'un point de vue politique, la dimension économique occupe une place secondaire. En effet, tandis que certains observateurs estiment que l'intensité du débat actuel sur l'immigration est manifestement liée à un mécontentement plus large de la population américaine vis-à-vis de l'État-Providence, plusieurs syndicats ont abandonné leur campagne de longue date contre l'immigration clandestine et se sont opposés à la Proposition 187. En outre, les électeurs californiens n'ont pas été particulièrement influencés par les arguments économiques, puisque seulement $32 \%$ de ceux qui ont voté en faveur de la Proposition 187 pensaient qu'elle permettrait à leur État d'économiser des millions de dollars et seulement $15 \%$ de ceux qui ont voté contre pensaient que cela coûterait à leur État des milliards de dollars en fonds fédéraux. Partis politiques, groupes d'intérêt et bureaucraties " Le marchand de la plaine de Cleveland expliqua en 1854: "Quand un Know Nothing ${ }^{36}$ veut se faire reconnaître, il ferme un oeil, forme un 0 avec le pouce et l'index et passe son nez à travers, cela signifie oeil-nez-0--1 know nothing ${ }^{37}$ - je ne sais rien." " 38 En 1994, le Parti Républicain a gagné pour la première fois depuis 1954 le contrôle des deux chambres au Congrès. Cette hausse du pouvoir des Républicains a largement contribué à la victoire de la Proposition 187 en Californie, où 78\% des électeurs Républicains ont voté en faveur de l'initiative, contre $36 \%$ des Démocrates et $62 \%$ des Indépendants. Néanmoins, la contradiction ne se trouvait pas entre les partis politiques mais en leur propre sein, et notamment au sein de la nouvelle majorité qui semblait être divisée sur un certain nombre de questions en matière d'immigration. Sur les thèmes qui devaient être choisis pour la plate-forme électorale par exemple, les membres du Parti Républicain oscillaient entre une position Know Nothing et une position libertaire extrême. Ainsi, on peut citer le président du Comité Ways and Means de la Chambre des Représentants qui affirmait: " sur le plan philosophique, je n'ai aucun scrupule à dire que nous ne 
sommes pas censés prendre en charge ceux qui ne sont pas devenus des citoyens " ${ }^{39}$, et le Maire de New York, Rudolf Giuliani, qui déclarait à la télévision : " si vous venez ici et que vous travaillez dur mais que vous êtes en situation irrégulière, vous faites partie de ceux que nous voulons avoir dans cette ville "40. Par ailleurs, le gouverneur Pete Wilson, même s'il avait érigé l'immigration clandestine en problème primordial et avait lié sa campagne électorale pour le siège du gouverneur à celle de la Proposition 187, distinguait généralement les immigrés en situation régulière, qui étaient les bienvenus, des immigrés clandestins. Le sénateur Alan Simpson, ainsi que Jack Kemp et William Bennett, s'étaient publiquement démarqués de la campagne en faveur de la Proposition 187. Kemp et Bennett avaient déclaré qu'elle était " politiquement imprudente et contraire à la tradition et à l'esprit de notre parti " et Simpson avait affirmé : " j'ai quelque problème à accepter qu'une personne âgée de sept ou de huit ans soit reconduite à la frontière quand nous n'avons pas les moyens d'expulser des criminels étrangers incarcérés dans nos prisons "41. Simpson avait proposé d'élaborer un texte législatif établissant un système d'identification sûr en vue de vérifier si une personne était autorisée à travailler et à bénéficier du Welfare mais d'autres conservateurs, comme William Kristol, " le gourou stratégique du GOP42 ", avaient dénoncé la recommandation faite par la Commission bipartisane sur la réforme d'immigration concernant la mise au point d'un fichier informatique, en la qualifiant d'" un grand pas vers le renforcement du pouvoir policier du gouvernement fédéral "43. Enfin, on doit noter qu'il y a une contradiction fondamentale mais relativement latente au sein des Républicains au sujet du lien entre immigration et culture. Il y a une " proposition conservatrice selon laquelle les États-Unis ne sont pas une abstraction idéologique mais une nation dotée d'une culture venant des îles britanniques "44. Francis Fukuyama avait pourtant répondu de manière catégorique que " Paul Gigot peut avoir raison ou tort en affirmant qu'un million de Zoulous travaillent plus dur qu'un million d'Anglais, mais un million de Taïwanais apportent certainement avec eux des structures familiales et entrepreneuriales plus fortes "45. En d'autres termes, Murphy Brown et Ice-T qui, à l'opposé des immigrés, ne travaillent pas dur et divorcent, sont responsables de la dégénérescence des structures familiales et de la culture américaine. Si le Parti Républicain parvient à trouver une formule qui dépasse ses différents clivages internes, il est probable que le Parti Démocrate le suive. Juste un mois après sa première élection, le président Clinton avait déclaré que l'immigration clandestine était un problème majeur et, sans doute, il avait tenu compte des 167 votes $^{46}(60 \%$ des votes nécessaires à l'élection d'un président) des six États engagés dans un combat juridique et diplomatique contre le gouvernement fédéral en demandant le remboursement des frais occasionnés par la présence des clandestins sur leur territoire. Le vice-président avait proposé un programme de démographie et d'environnement dont l'esprit n'était pas incompatible avec la campagne visant à limiter l'immigration aux États-Unis. Un des sénateurs démocrates de l'État de Californie, Diane Feinstein, s'est souvent rendue à la frontière près de San Diego et a lancé une campagne pour que soit instaurée à l'encontre des Mexicains une taxe de passage de la frontière, idée qui ne fut que brièvement soutenue par le Président Clinton. Enfin, depuis longtemps, le secrétaire d'État au Travail accorde une grande importance à l'expansion des emplois hautement qualifiés dans le secteur industriel et minimise la valeur des emplois sous payés du tertiaire, une position qui associe l'immigration clandestine à la stagnation industrielle. Même si, généralement, la communauté hispanique de Californie vote massivement pour le Parti Démocrate et s'est opposée fermement à la Proposition 187 (77\% des 
électeurs hispaniques ont voté non), son influence politique est bien limitée à cause de l'absentéisme et du nombre élevé de jeunes qui ne sont pas électeurs. Ceci peut être constaté, par exemple, par l'écart considérable entre la place qu'elle occupe au sein de la population californienne (27\%) et le taux de sa participation au vote de la Proposition $187(10 \%)$. Une dernière dualité de la politique nationale sur l'immigration relève de l'écart entre une hyperactivité législative et une paralysie bureaucratique. Efrem Zimbalist Jr., Tom Clancey, Top Gun et les Intouchables doivent probablement tous une part de leur popularité pour avoir été associés à des institutions comme le FBI, le Pentagone, le Trésor Public et la CIA, en dépit de Aldrich Ames, de WacoTexas, et d'autres cauchemars pour tous ceux qui sont chargés des relations publiques. L'INS n'est mentionné que dans deux émissions télévisées, Coneheads et Green Card: la première est une satire qui passe en direct à la télévision tous les samedi soirs, où l'on voit des extraterrestres bien respectables se faire poursuivre en vain par le directeur d'un service régional de l'INS ; la seconde raconte un mariage blanc qui tourne en une histoire romantique mais qui est contrarié à cause de l'examen approfondi effectué par les agents de l'INS. Plus sérieusement, le New York Times a publié pendant une semaine une série de longs articles où il accusait l'INS d'incompétence (impossibilité de les joindre au téléphone), de corruption (les employés accepteraient des pots-de-vin pour délivrer des cartes de séjour), d'insensibilité (des files d'attente interminables devant des bureaux où sont délivrés les différents documents requis) et de négligence vis-à-vis des criminels (le faible taux de reconduite à la frontière des criminels étrangers) ${ }^{47}$, en concluant par une phrase assassine: " l'INS est totalement dysfonctionnel ". D'autres experts qualifient l'INS de " Sibérie bureaucratique ", qui n'a ni le budget ni la réputation nécessaire pour attirer des gens talentueux embauchés par d'autres agences, et le décrivent comme une agence déchirée par des rivalités internes dues à ses multiples missions. Si nous voulons pourtant être justes, nous devons admettre que, dans un pays dépourvu d'un État fort et d'une tradition de hauts fonctionnaires, l'agence se trouve dans une position peu enviable, faisant l'objet de coups durs du fait de la négligence des autorités de tutelle qui l'ont bien délaissée. Le Congrès l'a toujours peu subventionnée : par exemple, en 1924 il n'avait affecté à la Border Patrol (qui dépend de l'INS) que 45 agents pour surveiller 8000 miles de frontière. Elle dispose actuellement de 284 officiers chargés des reconduites à la frontière, qui doivent traiter environ 200000 cas de criminels étrangers souvent éparpillés dans diverses prisons. Élu en novembre 1992, le Président Clinton n'avait pas nommé de commissaire à l'INS jusqu'en juin 1993 et le Sénat n'organisera les auditions nécessaires qu'en septembre, de sorte que l'agence était restée sans directeur pendant presqu'un an. Plus tard, quand la nouvelle commissaire Meissner a suspendu, à cause d'un déficit budgétaire, le transfert au FBI des cartes avec les empreintes digitales des étrangers, le président du Comité de surveillance du Congrès lui a dit : " il y a certaines choses qui ne relèvent pas de votre pouvoir discrétionnaire. Ceci est l'une d'elles ". En 1994, le Congrès a considérablement augmenté le budget de l'INS, qui est passé de près de 1,5 milliards à 2,1 milliards de dollars, dont une grande partie a été attribuée à la Border Patrol en vue de renforcer les nouvelles opérations de " défense de l'avant " menées à la frontière avec le Mexique, à El Paso et à San Diego. Au total, la Border Patrol a déployé 1000 agents supplémentaires à la frontière, dont environ la moitié a été embauchée et le reste détaché. Les journalistes continuent à dire que " la mauvaise gestion budgétaire a laissé l'agence à court de personnel, sous-équipée, s'accrochant malgré tout à défendre son travail contre l'immigration clandestine notamment dans ses bureaux situés sur le 
terrain "48. Aucune attention n'a été accordée aux circonstances atténuantes mentionnées par les officiers de l'agence, qui déclarent qu'il est difficile de digérer une grande, brusque et inattendue infusion d'argent tout en subissant une profonde réorganisation. On a accordé peu d'attention à l'arrière-plan législatif des opérations de l'agence, comme la législation perverse en matière de sanctions à l'encontre des employeurs, qui, jadis, distinguait entre l'" hébergement " illégal et l'emploi (Texas Proviso de 1952) et qui, maintenant, ne demande aux employeurs que d'effectuer un contrôle rapide des documents qui leur sont présentés (l'héritage de l'lRCA de 1986). Fédéralisme et tribunaux " Il est rare qu'une question politique émerge sans qu'elle ne se constitue, tôt ou tard, en question judiciaire "49. Parmi les immigrants clandestins du pays, près de $40 \%$ résident en Californie, tandis que les autres sont répartis essentiellement dans cinq autres États. Néanmoins, la question de l'immigration a été contrainte de passer par le mécanisme fédéral du système américain. Les relations entre Washington et les États ont leurs propres qualités pendulaires, oscillant entre une synergie souple et une confrontation ouverte. Dans le premier cas, on peut citer les restrictions prises à l'encontre des Asiatiques vers le tournant du siècle. Ainsi, en 1870, le Congrès avait modifié la loi américaine de 1790 sur la nationalité en vue d'accorder le droit de naturalisation aux personnes d'origine africaine mais, en même temps, il avait privé les Asiatiques de ce droit (ainsi que les personnes originaires de l'Inde puisque dans un arrêt rendu en 1923 par la Cour Suprême, l'arrêt US v. Bhagat Singh Thind, il avait été énoncé que les Hindous n'étaient plus considérés comme appartenant à la race blanche). N'ayant pas le droit de légiférer directement en matière d'immigration, l'État de Californie avait adopté en 1913 l'Alien Land $\mathrm{Law}^{50}$, qui interdisait aux étrangers ne remplissant pas les conditions pour obtenir la nationalité américaine de posséder des terres en Californie ${ }^{51}$. Le gouvernement fédéral exerce ses prérogatives en matière d'immigration dans le but de maintenir les États soit dans une position de subordination soit dans une position d'exclusion totale. Cela étant, bien que la Cour Suprême ait déclaré en 1976, dans l'arrêt Decanas v. Bica, qu'elle " n'avait jamais soutenu que tout acte législatif d'un État était un règlement de l'immigration et pouvait avoir le droit de préemption ", elle a pourtant confirmé une ordonnance californienne, qui interdisait aux employeurs de faire travailler des étrangers en situation irrégulière si cela avait un impact négatif sur la main d'oeuvre locale. La législation adoptée par le Congrès en 1986 et en 1990 a probablement élargi la portée des actions des États, qui peuvent avoir le droit de préemption, comme par exemple les lois locales sur l'emploi ${ }^{52}$. Quant à la Proposition 187, le blocage initial de son application par la Cour reposait sur la présomption que, même en tenant compte des règlements adoptés par la législation locale, elle contenait des mesures incompatibles avec la loi fédérale. Selon Mariana Pfaelzer, juge à la Cour du district de Los Angeles, " même si on agrafait ces règlements à chaque section de la Proposition 187, cela ne marcherait pas "53. Dans son arrêt, Pfaelzer a affirmé que la Proposition 187 représentait " un projet de régulation de l'immigration " qui empiétait sur les prérogatives du gouvernement fédéral en la matière "54. De même, la tentative de l'État de Californie d'intenter un procès contre le gouvernement fédéral pour le remboursement des frais occasionnés par l'éducation, l'incarcération et l'hospitalisation des clandestins a été rejetée par la Cour du district de San Diego, en partie à cause des cas précédents qui ont immunisé le gouvernement fédéral contre ce type de poursuites ${ }^{55}$. Le Président Clinton et le Congrès semblaient être prêts à accorder davantage de subventions à certains États lourdement endettés, mais il faut rappeler que la responsabilité publique en matière du coût d'incarcération 
des clandestins, qui avait été formellement établie en 1986, n'a jamais été appliquée. Entre-temps, le voyage qu'avait effectué le gouverneur Wilson jusqu'au Capitol Hill, espérant obtenir une certaine réparation par le Congrès, s'était déroulé dans des conditions meilleures que ses voyages antérieurs. Ceci était dû à l'augmentation du nombre des Républicains au Congrès et à la modération des demandes du gouverneur, lesquelles avaient baissé de 2,3 milliards de dollars en 1994 à 600 millions de dollars en 1995. Et, en fin de compte, son voyage avait plutôt suscité " de la sympathie mais n'avait pas donné lieu à une aide financière " ${ }^{56}$. Bref, on peut dire avec Jeffrey Rosen, " qu'au fond, la Proposition 187 était une question liée au fédéralisme "57. Les manoeuvres politiques et les poursuites juridiques relatives à la Proposition 187 ont pris plus d'importance après la fin plutôt qu'au cours de la campagne en sa faveur. Au coeur du débat se trouvait l'arrêt Plyler v. Doe, rendu en 1982, où la Cour Suprême avait annulé une loi texane qui interdisait la scolarisation des enfants des clandestins dans les écoles publiques. La Cour avait estimé qu'une telle loi entraînerait la formation d'une classe défavorisée et que, moralement, il était injuste de punir les enfants pour des crimes commis par leurs parents ${ }^{58}$. Cette décision avait été prise à une faible majorité (cinq contre quatre) et avait nécessité de nombreuses enquêtes préliminaires ; cinq des juges avaient été depuis remplacés, mais il en restait encore deux ayant voté pour et deux ayant voté contre. Le blocage de la Proposition 187 en Californie s'appuie aussi, voire même plus, sur l'arrêt Plyler v. Doe que sur le droit de préemption de la loi fédérale, et la fin de la partie est loin d'être connue. Les juristes qui soutiennent la Proposition 187 admettent que l'initiative comprend, certes, certaines phrases potentiellement problématiques mais estiment que l'existence d'un " intérêt d'État " pourrait être prouvée, notamment après que le GAO et l'Urban Institute aient montré que le coût imposé à l'État de Californie s'élevait à des milliards de dollars. Tout en s'opposant au " caractère nativiste " de la Proposition 187, Jeffrey Rosen avait affirmé dans le New Republic que " l'effondrement des arguments de Brennan (Juge à la Cour Suprême) au cours de la dernière décennie pourrait convaincre les juges de casser, dès qu'ils auront l'occasion, l'arrêt Plyler. En effet, cet arrêt doit être cassé : dans l'arrêt Casey sur l'avortement, les juges O'Connor, Kennedy et Souter avaient indiqué que les arrêts constitutionnels marquants devenaient très vulnérables quand des changements ultérieurs mettaient en question leurs prémisses ". Discriminations " Les hindous sont trop bruns pour pouvoir voter ici "59. L'étude classique de Gunnar Myrdal sur " la question noire " commence par l'argument que la discrimination raciale fait partie intégrante de " l'ensemble des problèmes de la civilisation américaine et ne peut être traitée de manière isolée " ${ }^{60}$. Cela dit, dans certains cas, les questions de race et d'immigration ont été traitées plutôt indépendamment l'une de l'autre car, incontestablement, le racisme n'a pas toujours été lié à la couleur de la peau. Par exemple, le parti des Know Nothings était farouchement opposé à l'entrée des migrants européens catholiques aux États-Unis, surtout s'ils étaient d'origine allemande ou irlandaise. Bien que ce Parti n'ait pas survécu à la Guerre Civile, ses objectifs ont été en partie réalisés grâce aux lois sur les quotas nationaux adoptées dans les années vingt. Afin de réduire au minimum le nombre de migrants venant de l'Europe du Sud et de l'Est, dans le calcul de ces quotas on avait pris comme base les flux de 1890, car l'immigration en provenance de cette partie de l'Europe n'avait eu lieu que pendant la période 1890-1910. Dans ce cas, la xénophobie était, en partie au moins, dissociée de la couleur de la peau. Inversement, les modifications de la loi sur l'immigration, adoptées en 1965, dans le contexte de la campagne pour les droits civiques, c'est-à-dire la même 
année de l'adoption du Voting Rights Act, qui avait permis au ministère de la Justice de rendre majoritaires les districts minoritaires des sept États du Sud, entraînant ainsi un changement profond de la composition ethnique des migrants aux États-Unis, n'avaient pas d'impact ethnique et racial important. Dans ce cas aussi, la libéralisation (ou la déracialisation) de l'immigration était, au moins en partie, dissociée de la couleur de la peau. L'histoire des communautés noire et asiatiques aux États-Unis montre, par contre, l'existence d'un lien pratiquement étroit entre le racisme et l'immigration. Les Noirs étaient manifestement une sorte de réfugiés à l'envers, vivant dès le début du XVIIe siècle dans un climat de peur de persécution, qui était bien justifiée. Les Asiatiques avaient commencé de venir en Californie vers le milieu du XIXe siècle et, comme nous l'avons déjà indiqué, la loi de 1870 sur les droits civiques, qui avait rendu illégale la discrimination raciale à l'encontre des Noirs, avait paradoxalement permis le transfert de celle-ci sur les Asiatiques. Ainsi, la Cour Suprême avait entériné le Chinese Exclusion Act malgré son caractère exclusionniste, fondé sur la race, en affirmant que " le pouvoir législatif du Congrès n'est subordonné à aucun pouvoir " (par conséquent, même pas à la Constitution) ${ }^{61}$. La Cour avait également donné son feu vert au Geary Act de 1892, qui obligeait les Chinois déjà présents sur le territoire américain de se faire enregistrer, ce qui, au fond, avait pour but de renforcer l'exclusion des nouveaux entrants. Dans les années vingt, la Cour avait estimé que les migrants originaires de l'Inde et des Philippines tombaient sous le régime applicable aux Asiatiques, rappelant par là l'esprit du titre du Literary Digest " les hindous sont trop bruns pour pouvoir voter ici ", d'autant plus que, dans leur majorité, les Indiens de Californie étaient des Sikhs ou des musulmans plutôt que des Hindous. Ces lois qui empêchaient le mélange des races n'ont été abolies qu'après la Deuxième Guerre mondiale. À ces victimes de la discrimination se sont ajoutés, de manière moins systématique, les Mexicains et les " Américano-Mexicains ". Ceux-ci sont devenus victimes au XIXe siècle, suite à certains arrêts, tels que l'arrêt Botiller v. Dominguez, où " la protection de la propriété privée, garantie par le Traité de Guadalupe Hidalgo, fut essentiellement invalidée "62. Leur victimisation continua au XXe siècle, suite à des incidents de violence, comme les émeutes urbaines de Zoot Suit, à des accords du type Jim Crowe et à des pratiques d'exploitation de la main d'oeuvre. Pourtant, à la suite de la guerre américanomexicaine, les Mexicains qui vivaient dans les territoires annexés étaient, au moins en principe, invités à déposer une demande de naturalisation et ne tombaient pas sous les Alien Land Laws applicables aux autres " bruns ". En ce qui concerne cette dernière restriction, Karen Leonard a révélé que certains Indiens originaires de la région de Punjab, vivant à l'Imperial Valley, avaient pu garder de facto le contrôle de leurs biens fonciers soit en les confiant à des banques, dont une fut d'ailleurs appelée la banque hindoue, soit en les transférant à leurs épouses mexicaines. Durant les trente dernières années, les Cubains ont fait l'objet d'un statut de réfugié bien tolérant, codifié par un ensemble de textes législatifs, adopté en 1966, qui ne les obligeait pas à prouver l'existence d'une crainte de persécution individuelle et qui leur offrait le statut de résident permanent s'ils avaient séjourné pendant un an et un jour aux États-Unis. Ces dispositions ont été sévèrement critiquées au Congrès par le Black Caucus ${ }^{63}$, qui a également mis en cause le rapatriement sommaire par les gouvernements Bush et Clinton de 30000 Haïtiens ainsi que le rejet d'environ 94\% des 60000 demandes d'asile déposées de 1992 jusqu'au milieu de $1994{ }^{64}$. Le New Yorker du 10 janvier 1994 qualifia le changement d'attitude de Clinton vis-à-vis des Haïtiens d'" acte le plus scandaleux de sa première année au pouvoir ". Bref, on peut dire que la communauté hispanique se 
trouve quelque part entre la tentation raciste et l'engagement d'offrir à tous des chances égales, à savoir entre les deux composantes du " dilemme américain ". Par conséquent, le caractère mixte des messages de la Proposition 187, élaborée dans un État caractérisé par la coexistence des communautés asiatiques, hispanique et noire, n'a rien d'étonnant. Malgré l'avis de Bill Ong Hing, spécialiste des questions d'immigration asiatique, selon lequel " pour la plupart des gens l'aspect économique est superficiel, l'enjeu sous-jacent est la race ", les communautés noire et asiatiques ont pratiquement voté identique à la Proposition 187 (47\% de oui). Selon Peter Skerry, " en ce qui concerne l'accès aux HLM, aux écoles et aux soins médicaux, les Noirs défavorisés se sentent ouvertement menacés par les immigrés. À cet égard, l'exemple classique est celui de l'hôpital Martin Luther King Jr. à Los Angeles, qui avait été construit suite aux émeutes urbaines de 1965 mais qui est actuellement rempli d'immigrants Latinos " ${ }^{65}$. Bien que son histoire ait été moins troublée par le " problème noir " et que les résultats du Latino National Political Survey aient clairement indiqué qu'elle s'opposait à une hausse éventuelle de l'immigration en provenance de l'Amérique Latine, et même si elle s'est déclarée fière d'être aux États-Unis, la communauté hispanique a majoritairement voté contre l'initiative ${ }^{66}$. En ce qui concerne l'opinion de l'ensemble de la communauté, des sondages ont révélé que 39\% des personnes ayant voté contre la considéraient comme raciste et anti-hispanique, mais l'argument qui revenait le plus souvent était que cette mesure " était mal rédigée et qu'elle ne pouvait pas résoudre le problème " ${ }^{67}$. Il semble alors que l'observation de Gunnar Myrdal sur les États-Unis des années soixante, selon laquelle " les préjugés constituent encore une attitude courante mais le racisme comme idéologie compréhensive n'est soutenu que par une minorité ", reste encore valable pour la Californie des années quatre-vingt-dix. Et pourtant, le lien entre préjugés ou racisme et immigration risque de perdurer, voire se renforcer, au fur et à mesure que les ÉtatsUnis avancent vers le XXIe siècle. Les chiffres et les images font de la Californie le précurseur d'une nouvelle tendance. Les projections démographiques indiquent qu'en l'an 2002 la majorité de la population ne sera plus blanche. Des villes comme Monterey Park, où la communauté asiatique représente à présent $57 \%$ de la population contre $3 \%$ il y a trente ans, vont se généraliser. Réels ou fictifs, les émeutes urbaines de Los Angeles de 1992 et le film Bladerunner, qui décrit Los Angeles en 2019, sont un mauvais présage pour le futur. Des articles sur l'immigration qui adoptent apparemment un point de vue neutre contiennent en fait des arguments qui, consciemment ou non, se rapprochent des idées racistes, comme par exemple: " les Anglos qui demandent pourquoi doivent-ils apprendre l'espagnol entreront au XXIe siècle à l'arrière du train "68. La nouvelle proposition attaquant l'affirmative action, qui est présentée comme l'enfant de la Proposition 187, a déjà placé la race au coeur de la question. Celle-ci résulte d'une multitude de facteurs complexes, incluant une culture dominante dont l'inconscient collectif et le sens de l'histoire ne se réveillent que les jours fériés, et promet de ranimer la question la plus émotionnelle de l'histoire de ce pays. Ainsi, suite à un incident déclenché dans la Faculté de Droit de l'Université de Berkeley, à cause de la diffusion de tracts favorables à la nouvelle " initiative des droits civiques ", le Chancelier de l'Université n'a pas caché son indignation en s'exclamant: " Plus je deviens âgé, plus je suis convaincu que le problème racial occupe une place centrale dans la société américaine. Les questions qui lui sont liées ne disparaissent jamais... Il me semble que le melting-pot va inévitablement éclater "69. L'aspect international " Je n'accepte aucune contrainte administrative et je ne connais rien de Rio Grande. " 
Commandant Langhorne, $1917^{70}$. La dynamique internationale en matière d'immigration et d'autres questions liant le Mexique à la Californie est si compliquée qu'un théoricien de la " California-Mexico connection " a abouti à la conclusion que celle-ci " ne pourrait jamais atteindre un niveau d'intégration élevé " mais que " rien ne nous permet de suggérer qu'elle se désintégrera... Il y a trop d'éléments en jeu qui sont étroitement liés entre eux et dépendants de divers centres de prise de décision... "71. On peut voir l'impact de cette diversité politique sur les lobbies mexicains au Capitole, sur la sensibilité du gouvernement mexicain au pouls politique de la communauté mexicano-américaine de Los Angeles, sur le développement en Californie d'une politique étrangère focalisée sur les bords du Pacifique et sur l'établissement d'une relation spéciale entre San Diego et Tijuana. Le résultat est l'existence simultanée d'un conflit et d'une coopération, allant de la coopération étroite, établie au début du XXe siècle entre les agents américains et mexicains afin d'assurer l'application des lois sur la neutralité, jusqu'à la fermeture virtuelle de la frontière, suite à l'assassinat, en 1985, d'un agent de la DEA. Les politiques d'immigration sont déterminées par le push-pull des dynamiques transfrontalières intermestiques ${ }^{72}$. Du côté mexicain, une des conséquences est l'adoption d'un ensemble de mesures contradictoires, qui à la fois favorisent et défavorisent la coopération avec les États-Unis en vue de réduire le flux de travailleurs (al oltro lado) à la frontière. En cas de non coopération, le Mexique profite d'une nette hausse des envois de fonds des émigrés, estimés à 3,2 milliards de dollars en 1990 , ce qui est légèrement inférieur aux revenus provenant du tourisme (3,4 milliards de dollars) mais nettement supérieur aux investissements étrangers directs (2,6 milliards de dollars) ${ }^{73}$. Par contre, une attitude plus coopérative a été adoptée par Salinas, lors de la campagne en faveur de l'ALENA, et par Zedillo, pour obtenir le soutien américain au peso. Toujours dans le sens de la coopération, le Mexique a adopté certaines mesures de contrôle du passage des ressortissants des pays tiers qui traversent son territoire pour entrer aux États-Unis, comme, par exemple, l'interception dans les aéroports des clandestins venant du Moyen-Orient, le renforcement des contrôles de la frontière du Sud en vue de réduire les flux venant de l'Amérique centrale ainsi que le contrôle des frontières maritimes de la côte Pacifique pour renvoyer les boat-people chinois, opérations pour lesquelles les États-Unis lui ont versé environ un demi million de dollars. On peut citer également le déploiement par le Mexique, tout au long de la frontière américaine, des unités de police spéciale (Grupo Beta), créées pour lutter contre les activités criminelles liées à l'immigration, ainsi que son engagement à lutter contre la fabrication de fausses cartes d'identité à l'intérieur du pays et à augmenter ses efforts dans la lutte contre les réseaux de contrebande. La conséquence la plus significative de cet ensemble de mesures est probablement l'aspect public de l'accord de coopération avec le voisin du Nord qui, à plusieurs occasions, comme en 1917, a préféré fermer les yeux sur ses propres frontières. Sécurité " L' usage de l'opium ainsi que la morphinomanie et la cocaïnomanie se répandent rapidement au sein de la population blanche non seulement en Californie mais dans tous les ÉtatsUnis... Pour cette unique raison, l'immigration chinoise doit être arrêtée et nous devons faire un effort pour éloigner les coolies qui se trouvent déjà ici "74. La nécessité de reconceptualiser la sécurité dans l'ère post-bipolaire est devenue une priorité. Pendant près d'un demi siècle, obsédés par l'éventualité d'une attaque nucléaire imprévue, les universitaires et les pouvoirs publics ont adopté une définition restrictive des menaces qui est, de nos jours, remplacée par une nouvelle approche frénétique des dangers virtuels, allant des obsessions classiques en matière militaire aux problèmes 
d'environnement, à la mafia et aux autres opérations criminelles transnationales, au trafic de stupéfiants, aux guerres du commerce agricole, au terrorisme et, en ce qui concerne notre propos, à l'immigration clandestine. Celle-ci a été présentée comme une menace nouvelle et potentiellement importante en raison du volume des flux migratoires prévus (mesurés comme s'il s'agissait de flux aquatiques), de la réévaluation importante des thèmes de société et de culture en matière de sécurité internationale (propositions théoriques de la " sécurité sociétale " et du " clash des civilisations ") et des liens entre immigration clandestine et autres dangers (trafic de stupéfiants et terrorisme de la cinquième colonne en particulier). Chaque composante de cet ensemble de menaces semble créer plus de problèmes conceptuels qu'elle n'en résout. Jusqu'à présent, par exemple, les États-Unis n'ont pas été confrontés à des flux massifs de réfugiés engendrés par le nouveau désordre mondial. Le rapport de $1993 \mathrm{du}$ Comité américain pour les réfugiés révèle qu'aux États-Unis il y a 100000 réfugiés et demandeurs d'asile ayant besoin de protection et/ou d'assistance, contre 5,7 millions en Afrique, 5,6 millions au Moyen-Orient, 3,3 millions en Europe et 2,7 millions en Asie $^{75}$. De même, les estimations contradictoires sur le nombre des clandestins chinois, plus d'un million selon le Survival et près de 27000 selon l'INS, rappellent étrangement les pratiques qui étaient en vigueur pendant la Guerre Froide, celles qui exagéraient le problème en surestimant le nombre et le niveau de préparation des troupes du Pacte de Varsovie $^{76}$. Quant aux paradigmes nouveaux ou réactualisés de sécurité internationale, qui mettent l'accent sur la société et la civilisation plutôt que l'État, la diatribe de Huntington sur les " frontières ensanglantées " de l'islam et les transferts d'armes à travers la " connexion islamo-confucéenne " est particulièrement superficielle. Elle nie complètement l'importance de l'enrichissement mutuel des sociétés contemporaines, elle est incapable d'expliquer la coalition politique obtenue par les États-Unis lors de la guerre du Golfe et elle omet de mentionner la position ouvertement dominante des États-Unis dans le bazar mondial de l'armement. Enfin, même si ces analyses qui lient l'immigration clandestine et les autres dangers dans un " continuum de menaces " doivent incontestablement faire l'objet de réflexions plus approfondies, les prétendues connections entre l'immigration et les risques sécuritaires semblent être plutôt tendancielles, fortuites ou, au mieux, faibles. Par exemple, il est vrai que la connexion mexicaine est passée de ce qu'Elaine Shannon ${ }^{77}$ désignait comme la " frontière de la marijuana ", dans les années soixante-dix, à celle d'une plus grande variété de stupéfiants dans les années quatre-vingt-dix. Elle est impliquée, par exemple, dans le trafic de cocaïne colombienne dirigé à nouveau des Caraïbes. En outre, l'ALENA rendra encore plus difficile son contrôle, puisque le trafic transfrontalier sera accéléré afin de satisfaire les besoins d'un commerce en pleine croissance. Mais la seule connection significative entre les immigrants clandestins et les cargaisons de stupéfiants en provenance du Mexique est que tous les deux traversent la même frontière et suivent pratiquement le même itinéraire ; les immigrants ne possèdent pas (et ne consomment pas) d'importantes quantités de stupéfiants. En 1994, la Border Patrol du secteur de San Diego a interpellé 450000 immigrants clandestins, dont seulement $95(0,02 \%)$ ont été arrêtés pour possession de stupéfiants ${ }^{78}$. Comme pour la connection entre le crime organisé et l'entrée d'immigrants clandestins, suggérer que ce trafic constitue une menace sécuritaire revient à confondre l'arbre avec la forêt. Finalement, en ce qui concerne la connection entre l'immigration clandestine et le terrorisme, le World Trade Center et les procès qui ont eu lieu suite à cet attentat ont clairement montré qu'il y avait un lien entre les irrégularités procédurales, commises dans l'application de 
la législation sur l'immigration, et les risques de menace à la sécurité nationale. Par exemple, Mahmud Abouhalima, un des principaux acteurs de l'attentat, n'avait pas quitté les États-Unis après l'expiration de son visa touristique et avait régularisé son statut en profitant frauduleusement d'une amnistie : il avait déclaré qu'il avait travaillé dans une ferme en Caroline du Sud alors qu'il était chauffeur de taxi à New York ${ }^{79}$. Aujourd'hui, il semble que l'association entre l'islam et le terrorisme ne soit plus exceptionnelle mais soit devenue la nouvelle image des personnes originaires $\mathrm{du}$ Moyen-Orient. Cette simplification est particulièrement manifeste lorsque nous employons des expressions comme " fondamentaliste islamiste " ou " terroriste arabe ", lesquelles, dans l'imaginaire public américain, sont devenues quasiment identiques. De même, Saddam Hussein et l'Ayatollah Khomeini représentent la version négative des frères jumeaux du genre Franklin D. Roosevelt et Churchill, et les terroristes arabes fanatiques dans le film True Lies transportent des têtes nucléaires en les dissimulant, bien évidemment, dans des objets anciens d'origine persane. Si les efforts du gouvernement pour développer des techniques de lutte contre le terrorisme sont nécessaires, le spectre de la cinquième colonne doit être expliqué à la société américaine en évitant de reproduire dans l'ère post-bipolaire l'internement discriminatoire des Japonais-Américains pendant la Deuxième Guerre mondiale et la campagne menée à l'encontre des Mexicano-Américains soupçonnés de trahison pendant la Première Guerre mondiale. Peu ou guère connue, cette dernière était la réaction à un plan mexicain élaboré par Carranza, appelé le Plan de San Diego, d'après le nom d'une petite ville texane, incitant les Mexicano-Américains à se révolter et à massacrer tous les hommes adultes d'origine britannique, qui a donné lieu à une douzaine d'incursions aux États-Unis vers 1915-16. Mais, finalement, " les principales victimes furent des centaines de Mexicano-Américains lynchés par les Texas Rangers et par les autorités locales "80. En fin de compte, il se peut que le lien le plus convaincant entre sécurité et immigration soit un lien historique, où, poussés par la compétition géopolitique, les empires américain et européen ont généré ou influencé des flux migratoires venant du tiers-monde. Les programmes de guestworker ont toujours été le reflet des rapports " néo-coloniaux ", qu'il s'agisse des Algériens en France ou des braceros mexicains aux États-Unis. De même, les flux de réfugiés ont été le produit d'un ensemble de prétentions stratégiques globales, d'objectifs idéologiques et de compassion mesurée, comme ce fut le cas des Cubains en Floride et des Vietnamiens en Californie. Plus récemment, le réseau qui a planifié les attentats de New York aurait été entraîné, financé et soutenu par des agences américaines impliquées dans la campagne contre la présence de l'Union soviétique en Afghanistan. L'immigration en provenance des colonies a aussi joué un rôle important dans la consolidation de l'indépendance du tiers-monde. Aux États-Unis, par exemple, " l'exclusion et l'indépendance ont été étroitement liées " lors du débat des années trente qui a abouti à l'adoption, en 1934, du Tydings McDuffie Act, qui a rendu aux Philippines leur indépendance après la Deuxième Guerre mondiale ${ }^{81}$. En France, l'idée d'une Algérie française entièrement intégrée était déjà discréditée avant même que de Gaulle ne prenne le pouvoir et ne prononce le mot " foutue ", en raison de la réalité démographique signalée par Raymond Aron, selon qui, neuf millions de musulmans se reproduisaient beaucoup plus vite que cinquante millions d'européens. En Grande-Bretagne, l'immigration était devenue l'atout de la " décolonialisation " menée par les Tories dans les années soixante. Le fameux discours d'Enoch Powell, Rivers of Blood, avait mis fin à la nostalgie de l'empire ${ }^{82}$. Conclusion " Les deux hommes, Gibreelsaladin et 
Farishtachamcha, condamnés à cette incessante chute angélico-diabolique, ne se sont pas rendus compte du moment du commencement du processus de leur transmutation "83. La Proposition 187 peut marquer le début d'une transformation de la politique américaine d'immigration, mais il reste à savoir qui développera une auréole (à l'instar de Farishta, irrespectueux et exhibitionniste, figurant au début du roman de Rushdie) ou des cornes et des sabots (à l'instar de Chamcha qui, quand il était jeune à Bombay, avait déjà anticipé la règle de Tebbit et avait applaudi l'équipe de cricket anglaise). Mais il est clair qu'il n'y a pas de synthèse révolutionnaire ni de compromis hâtifs en préparation. L'immigration clandestine est par définition problématique. En modifiant légèrement la formule de Churchill, on peut dire que la règle de droit est la pire forme d'autorité, à l'exception de toutes les autres. Cesar Chavez, le chef héroïque des Latinos au sein $\mathrm{du}$ United Fruit Workers, considérait dans les années soixante-dix l'immigration clandestine comme " un problème grave " et dénonçait régulièrement les clandestins aux autorités fédérales. Le taux élevé de fraudes commises lors du programme de régularisation $\mathrm{SAW}^{84}$, en 1986, où, au lieu des 250-350 000 demandes prévues, avaient été déposées 1275000 demandes, avait mis de l'huile sur le feu ${ }^{85}$. Enfin, l'estimation selon laquelle $15 \%$ de la population américaine d'origine étrangère serait né à l'étranger mais que $30 \%$ du flux migratoire annuel vers le pays serait en situation irrégulière ne suggère pas que le problème ait été résolu. D'autre part, l'immigration clandestine doit être considérée comme un problème en soi ayant sa propre histoire. Il est exagéré de la considérer comme un problème ayant des conséquences graves, notamment sur la situation économique du pays en général. Les 600 millions de dollars que le gouverneur de l'État de Californie souhaite se faire rembourser par le gouvernement fédéral, en estimant qu'ils correspondent à la somme que dépense son État à cause des clandestins, correspondent à 20 dollars par habitant, soit $1 \%$ du budget de l'État ou le coût marginal de fabrication d'un bombardier B-2 selon les estimations du fabricant (sans moteurs, accessoires, équipement de soutien et carburant). Le seul danger grave est que les rhétoriques transforment cette question complexe en une question démagogique dotée d'un caractère racial. Certes, le slogan " sauvons notre État " s'inscrit dans une logique de simplification propre à toute campagne, mais des déclarations comme celles faites par les auteurs de la Proposition 187, selon lesquels, " nous n'avons pas les moyens pour éduquer, soigner, incarcérer et indemniser tous les immigrants clandestins du monde ", et l'insinuation faite par un sénateur de l'État de Californie qu'en cas d'adoption de la Proposition 187 les Latinos seraient obligés de porter sur eux une pièce d'identité, poussent cette mesure vers la démagogie et le racisme. La composition démographique de la Californie a été largement multi-ethnique et multiraciale dès la création de l'État au XIXe siècle, comme en témoignent les noms des premiers bourgs et camps miniers, tels que Sonora Bar, Chili Camp, Dutch Flat, Irishtown, Italian Gulch, Portuguese Flat, Norwegian Mine, Swiss Bar et Chinee Camp. Cette diversité a été renforcée tant par le push-pull de l'économie internationale et les liens établis de manière fortuite avec le reste du continent que par la campagne contre le communisme à travers le monde. Les résidents des États-Unis ne doivent pas célébrer la diversité mais ils doivent la tolérer ; sinon, la seule alternative sera d'imiter la réponse de Khomeini à la dialectique de Rushdie. 


\section{NOTES}

1. Randy Willoughby est Professeur de Science Politique à l'Université de San Diego. L'auteur voudrait surtout remercier Al Sweedler et Mike Willoughby de l'avoir prudemment guidé et d'avoir été souvent en désaccord avec lui. Il est aussi reconnaissant à la James Irvine Foundation et à l'Université de San Diego pour leur soutien financier. Il remercie enfin Ana Evans et Ivani Vassoler de leur aide en matière de recherche.

2. Stuart Schram, Chairman Mao Talks to the Peop1e, New York, Pantheon, 1974. 3. Denise Dresser, " Exporting Conflict : Transboundary Consequences of Mexican Politics " in Abraham Lowenthal and Katrina Burgess (eds), The Ca1ifornia-Mexican Connection, Stanford University Press, 1993.

4. Kitty Calavita, " The Immigration Policy Debate" in Wayne Cornelius and Jorge Bustamante (eds), Mexican Migration to the United States, Center for US-Mexican Studies, University of California, San Diego, 1989, pp. 164 et 176, pp. 151-178.

5. Phillip Martin and Mark Miller, " European-American Immigration Convergence ", International Migration Review, vol. XXVIII, n 3, automme 1994, pp. 591-594.

6. Salman Rushdie, The Satanic Verses, Dover, The Consortium, 1988.

7. Dos Mouillés : Terme péjoratif employé pour désigner les Mexicains entrés clandestinement dans le territoire américain en traversant à la nage le Rio Grande/El Norte.

8. P. Martin and M. Miller, op. cit..

9. Didier Bigo, " The European Internal Security Field " in Malcolm Anderson and Monica den Boer (eds), Policing Across National Boundaries, London, Pinter, 1994.

10. Hedda Hopper à Charlie Chaplin. Cf. David Robinson, Chaplin : His Life and Art, New York, McGrawHill, p. 579.

11. Richard Dillon, Humbugs and Heroes : A Gallery of California Pioneers, New York, Doubleday and Company, 1970, p.190.

12. Jim Williams, Ellis Island and Statue of Liberty, San Francisco, American Park Network, 1992.

13. Jeffrey S. Passel and Michael Fix, " Myths About Immigrants ", Foreign Policy, été 1994, pp. 151-160.

14. A. M. Rosenthal, " Working in Tijuana ", New York Times, 21.02.1995.

15. Rick Shaugnessy, " Many Immigrants Demonstrate Flair for Creating Jobs ", San Diego Union-Tribune, 31.01.1995.

16. Eduardo H. Galeano, Century of the Wind, New York, Pantheon, 1988, p. 14.

17. Marcus Stern, " Foul-ups Keep Cash from INS in Field ", San Diego Union-Tribune, 05.02. 1995.

18. Robert Warren, Estimates of the Unauthorized Immigrant Population Residing in the United States, by country of Origin and State of Residence, October 1992, Washington DC, INS Statistics Division, 29.04.1994.

19. Rudy Murillo, statistiques de l'INS fournies à l'auteur en 1995.

20. Ashley Dunn, " Greeted at Nation's Front Door, Many Visitors Stay On Illegally ", New York Times, 03.01.1995. 
21. Rebecca L. Clark, Jeffrey S. Passel, Wendy N. Zimmermann and Michael E. Fix, Fiscal Impacts of Undocumented Alien, Selected Estimates for Seven States, Washington D. C., The Urban Institute, 16.09.1994.

22. Richard Parker and Louis Rea, " Illegal Immigration in San Diego County : an Analysis of Costs and Revenues ". Prepared for Senator William A. Craven, California State Senate, Special Committee on Border Issues, 1993, p. 71.

23. Daniel Wolf, " The Rea-Parker Study of Undocumented Alien Fiscal Impacts : How Accurate? ", Testimony to the Immigration Hearing and Public Forum of Bill Morrow Assemblyman, Oceanside, CA, 09.12.1993.

24. J. Passel et M. Fix, op. cit..

25. Roy Beck, " Right of Silence ? The GOP and Immigration ", Nationa1 Review, 11.07.1994, pp. 27-35.

26. Alejandro Portes and Robert Bach, Latin Journeys, Berkeley, University of California Press, 1985, p.77.

27. Manuel Garcia y Griego, " Policy-Making at the Apex : International Migration, State Autonomy and Societal Constraints " in Jorge A. Bustamante, Clark W. Reynolds, Raul A. Hinojosa Ojeda (eds), US-Mexico Re1ations : Labor Market Interdependence, Stanford University Press, 1993.

28. A. Dunn, op. cit..

29. David Hackett Fischer, Historians' Fa11acies, New York, Harper and Row, 1970, p. 61.

30. SANDAG ; L. Rea and R. Parker, op. cit..

31. Peter Nunez, Presentation to the San Diego World Affairs Council, 19.10. 1994.

32. George Borjas, The Economics of Immigration, mai 1994, unpublished paper.

33. J. Passel et M. Fix, op. cit., p. 158.

34. G. Borjas, op. cit., p. 46.

35. G. Borjas, op. cit., p. 43.

36. Membre du parti nationaliste et xénophobe du XIXe siècle, composé en majorité d'ouvriers qui se déclaraient menacés par la main d'oeuvre étrangère ainsi que de Sudistes qui étaient affaiblis par le développement économique du Nord du pays.

37. Jeu de mot sur I-know-O, (eye, nose, zero).

38. William Safire, Safire's Po1itica1 Dictionary, New York, Ballantine, 1978, p. 360.

39. Dick Kirschten, " Second Thoughts ", Nationa1 Journal, 21.01.1995, pp. 150-155.

40. R. Beck, op. cit..

41. D. Kirschten, op. cit., p. 151.

42. Grand Old Party, expression qui désigne le Parti Républicain.

43. D. Kirschten, op.cit., p. 154.

44. R. Beck, op. cit.

45. Francis Fukuyama, "Immigrants and Family Values ", Commentary, mai 1993, pp. 26-32.

46. Californie : 54 votes, New York : 33, Texas : 32, Floride : 25, New Jersey : 15, Arizona : 8.

47. Joel Brinkley, " At Immigration, Disarray and Defeat ", New York Times, 11.09.1994.

48. Marcus Stern, "White House Inflated Data on Migrants ", San Diego Union-Tribune, 28.01.1995.

49. Alexis De Tocqueville, Democracy in America, New York, Random House, 1945.

50. Loi sur la propriété foncière des étrangers. 
51. Bill Ong Hing, Making and Remaking Asian America through Immigration Policy, Stanford University Press, 1993.

52. David Weissbrodt, Immigration Law and Procedure, St Paul, West Publishing Co., 1992.

53. Kenneth B. Noble, " Court Deals Another Blow to Backers of Proposition 187 ", New York Times, 15.12.1994.

54. San Diego Union-Tribune, 20.01.199.

55. Idem, 14.02.1995.

56. Mark Z.Wilson Barabak, " Gets Sympathy, No Cash Commitment from US ", San Diego Union-Tribune, 02.02.1995.

57. Jeffrey Rosen, " The War on Immigrants ", The New Repub1ic, 30.01.1995, p. 26.

58. Bob Egelko, " Prop. 187 Doesn't Violate Due Process, State Says ", San Diego UnionTribune, 08.12.1994.

59. Titre extrait du Literary Digest de 1923, cité par Karen Leonard dans Making Ethnic Choices, Temple University Press, 1992, p. 55.

60. Gunnar Myrdal, An American Dilemma, New York, Pantheon, 1962, p. 75.

61. D. Weissbrodt, 1992, op. cit.

62. Richard Griswold del Castillo, The Treaty of Guadalupe Hida1go, Norman University of Oklahoma Press, 1990, chap. 7, p. 76.

63. Lobby des Noirs au Congrès.

64. The Economist, 16. 07. 1994.

65. Peter Skerry, " The Black Alienation ", The New Republic, 30.01.1995.

66. The Economist, 08.01.1993.

67. San Diego Union-Tribune, 09.11.1994 ; Nationa1 Journal, 18.06.1994.

68. Brae Canlen, " San Diego Burning ", Ca1ifornia Lawyer, août 1993, pp. 44-119.

69. B. Drummond Ayres, " Conservatives Forge New Strategy to Challenge Affirmative Action ", New York Times, 16.02.1995.

70. Citation extraite de Ethan A. Nadelmann, Cops Across Borders, University Park, Pennsylvania State University Press, 1993.

71. James Rosenau, " Coherent Connection or Commonplace Contiguity? " in Abraham Lowenthal and Katrina Burgess, op.cit., p. 30.

72. Mot-valise composé d'international et de domestic.

73. Fernando Lozano Ascencio, Bringing It Back Home, Center for US Mexican Studies, University of California, San Diego, 1993.

74. Terence Emmons, Around California in 1891, Stanford Portable, Stanford Book Series, 1991, p. 42.

75. Doris M. Meissner et al., International Migration Challenges in a New Era, New York, The Trilateral Commission, 1993.

76. Paul J. Smith, " The Strategic Implications of Chinese Emigration ", Surviva1, vol. 36, n 2, été 1994, pp. 60-77.

77. Elaine Shannon, Desperados, New York, Viking, 1988.

78. Marco Ramirez, statistiques sur la Border Patrol fournies par téléphone à l'auteur (23.02. 1995).

79. Richard Behar, " Muhammad the Red ", Time, 04.10.1993.

80. E. Nadelmann, op. cit.

81. B. Ong Hing, op. cit.

82. Miles Kahler, Decolonization in Britain and France, Princeton University Press, 1984. 
83. S. Rushdie, op. cit..

84. Programme SAW, " Seasonal Agricultural Worker " : Programme d'admission des travailleurs temporaires, adopté en 1986.

85. Wayne Cornelius, " From Sejourners to Settlers " in Jorge A. Bustamante, Clark W. Reynolds et al., op. cit., p. 167.

INDEX

Mots-clés : immigration, racisme, sécurité, frontières

Index géographique : Etats-Unis, Mexique 